\title{
THREE GIANTS - MEMBERS OF THE OPEN CLUSTER M 67
}

\author{
T.Mishenina ${ }^{1}$, V.Klochkova ${ }^{2}$, V.Panchuk ${ }^{2}$, N.Basak ${ }^{1}$, V.Kovtyukh ${ }^{1}$, S.Korotin ${ }^{1,3}$, A.Velichko ${ }^{4}$ \\ ${ }^{1}$ Astronomical Observatory, Odessa National University, 65014-UA Odessa, Ukraine \\ tmishenina@ukr.net \\ ${ }^{2}$ Special Astrophysical Observatory, Nizhnij Arkhyz, 369167, Russia \\ ${ }^{3}$ Crimean Astrophysical Observatory, Nauchny, 298409, Crimea \\ ${ }^{4}$ V. N. Karazin Kharkiv National University, Kharkiv, Ukraine
}

\begin{abstract}
We determined the atmospheric parameters and chemical composition of the three giants, which are members of the open cluster M 67. The high resolution spectra $(\mathrm{R}=60000)$ were obtained with the echelle spectrograph NES mounted on the 6-meter telescope of the SAO RAS Obtained variations in $\mathrm{Na}$ and $\mathrm{Zr}$ abundances are within the determination errors. The $\mathrm{Li}$ abundance suggest a depletion of $\mathrm{Li}$ in the course of stellar evolution. For studied giants, the iron abundances derived from the neutral and ionized iron lines have not shown any significant discrepancies. The $[\mathrm{Ba} / \mathrm{Fe}]$ values are close to the solar ones.
\end{abstract}

Keywords: stars: abundances - stars: atmospheres Galaxy: open clusters and associations: individual: M 67

\section{Introduction}

The open cluster M 67 (NGC 2682) was discovered by Johann Gottfried Koehler in 1779 and is one of the best studied open clusters (OCs). It is also one of the oldest known Galactic clusters with an estimated age of about 4.3 Gyr (Pietrukowicz, 2006), it lies at a distance between 800 and 900 pc away (e.g. Sarajendini et al., 2009), and has the reddening value $\mathrm{E}(\mathrm{B}-\mathrm{V})=0.041 \pm 0.004$ (Taylor, 2007). M 67 contains a high percentage of blue stragglers, stars which are bluer and more luminous than the main-sequence turn-off point of the cluster (Pribulla et al 2008); for this cluster, the turn-off occurs near $\mathrm{B}-\mathrm{V}=0.55$, and $\mathrm{V}=13.0$.

Our target stars, namely 84, 141, 151 (Fagerholm, 1906) show enhancement in CN band strengths and are 'clump' giants (Pagel, 1974). Carraro et al. (1996) evaluated the mass loss in M 67 based on the determinations of masses of the clump stars and RGB giants. Due to a small offset as d[B-V] $=0.01 \mathrm{mag}$, it was unfeasible to use the clump stars to infer the efficiency of mass loss along the RGB phase. They also reported that there was no evidence for a dependence of the mass-loss rate from RGB stars on metallicity.

Tautvaišienè et al. (2000) analyzed spectra of six core helium-burning 'clump' stars and three giants in M 67 to detect any signs of extra mixing associated with the $\mathrm{He}$ core flash. They claim that the metallicity of M67 is close to solar $([\mathrm{Fe} / \mathrm{H}]=-0.03 \pm 0.03$, the carbon is depleted by about $0.2 \mathrm{dex}$, nitrogen is enhanced by about $0.2 \mathrm{dex}$ and oxygen is unaltered. The mean $\mathrm{C} / \mathrm{N}$ and ${ }^{12} \mathrm{C} /{ }^{13} \mathrm{C}$ ratios are lowered to the values of $1.7 \pm 0.2$ and $24 \pm 4$ in the giants and to the values of $1.4 \pm 0.2$ and $16 \pm 4$ in the clump stars. The results obtained enabled to suggest that extra mixing of CN-cycled material to the stellar surface takes place after the He-core flash. Abundances of other elements in investigated stars were found to be close to the solar ones.

Our goal is to re-determine the parameters and chemical composition of the three clump stars of the M 67 cluster. The stars in question are shown on the Hertzsprung-Russell diagram for M 67 presented in Fig. 1; the V and B-V values have been adopted from Geller et al. (2015).

\section{Observations and radial velocities}

The main characteristics of the M 67 cluster stars, as well as the observational data, are given in the Tables 1, 2, respectively.

The spectral data have been obtained with the echelle spectrograph NES (Panchuk et al., 2006, 2017) mounted on one of the Nasmyth focus platforms of the 6-meter telescope of the SAO RAS. NES provides a resolving power of $\mathrm{R} \geq 60000$ within the wavelength region 3200 $10000 \AA \AA$. The reduction of stellar spectra, as well as the measurement of radial velocities VR, has been performed using the DECH20 software package (Galazutdinov, 1992).

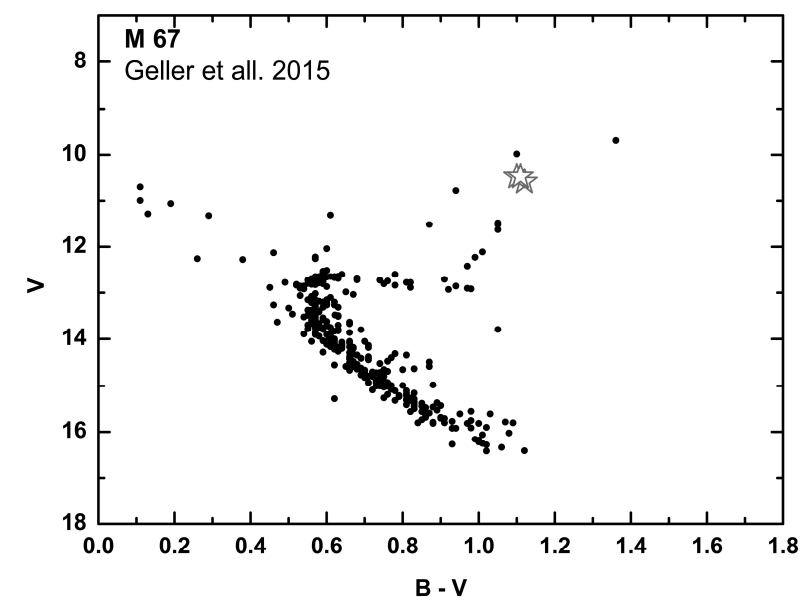

Figure 1: The H-R diagram for M 67; the V and B-V data have been taken from Geller et al. (2015). Clump giants marked as asterisks. 
Mathieu et al. (1986) have reported that the M 67 mean radial velocity $\mathrm{VR}=33.5 \pm 2.8 \mathrm{~km} / \mathrm{s}$ (for 65 cluster member stars). It can be seen that there is good agreement between our VR values and those obtained by other authors (Table 2).

\section{Parameter determination}

The effective temperatures $\mathrm{T}_{\text {eff }}$ were estimated by calibration of line-intensity ratios $-\mathrm{T}_{\text {eff }}$ (Kovtyukh et al., 2006); the results are presented in Table 3.

To determine the gravity log $g$ we used two methods: iron ionisation equilibrium (IE) and parallaxes (Pi). The obtained $\log g$ values are given in Table 4.

For two stars (namely, 84 and 141), we have obtained a noticeable difference in the value of gravity values, which is associated with use of two different methods. Further computations of elemental abundances have been performed using $\log \mathrm{g}$ derived under conditions of ionization equilibrium for iron.

It should be noted that when using the parallax, we encountered a certain problem with parallax measurements can result in different distances to objects, which in turn leads to different values of gravity. For example, the parallax for the star 84 is 0.00097 and corresponds to the distance to the object $1030 \mathrm{pc}$. For the star 141, the parallax is 0.00088 and the relevant distance is $1136 \mathrm{pc}$. For the star 151 the parallax was markedly different from those for the two other target stars: the parallax for 151 is 0.00240 ; that means the determined distance, which is $417 \mathrm{pc}$. The latter distance value differs significantly from the distances to this cluster estimated earlier (e.g. Sarajendini et al., 2009), as well as from the data obtained recently by Viani \& Basu (2017), who have reported that from the resulting isochrones that fit the M 67 cluster, the age range is between 3.6 and $4.8 \mathrm{Gyr}$ while the distance is between 755 and $868 \mathrm{pc}$.

Microturbulent velocity $V_{t}$ was determined from independence of an abundance $\log \mathrm{A}(\mathrm{Fe})$ from equivalent width $\mathrm{EW}$ for $\mathrm{Fe} I$ lines. The metallicity $[\mathrm{Fe} / \mathrm{H}]$ was adopted as the iron abundance determined from Fe I lines.

All the parameters, which we have obtained, are presented in Table 5.

\section{Chemical abundance determination}

The elemental abundances were determined using Castelli \& Kurucz's (LTE) models; the Kurucz WIDTH9 code was used for the LTE determination of $\mathrm{Si}, \mathrm{Ca}, \mathrm{Ni}$, $\mathrm{Fe}, \mathrm{Zn}, \mathrm{Y}, \mathrm{Zr}, \mathrm{La}, \mathrm{Ce}, \mathrm{Pr}, \mathrm{Nd}$ and Sm abundances; the modified latest version of STARSP (Tsymbal, 1996) was employed for the LTE determination of $\mathrm{Li}$ and $\mathrm{Eu}$ abundances; and the modified MULTI code (Carlsson, 1986; Korotin et al., 1999) was used for the NLTE determination of $\mathrm{O}, \mathrm{Na}, \mathrm{Al}, \mathrm{Mg}, \mathrm{Ca}$ and $\mathrm{Ba}$ abundances. The spectrum synthesis fitting of the $\mathrm{Mg}$ and $\mathrm{Na}$ lines to the observed profiles for 141 star is shown in Figs. 2, 3.

The elemental abundances $[\mathrm{El} / \mathrm{Fe}]$ as function of metallicity $[\mathrm{Fe} / \mathrm{H}]$ for the investigated stars are depicted in Fig. 4.
Table 1. The main characteristics of the target M67 giants

\begin{tabular}{|l|l|l|l|l|l|l|l|}
\hline Star & star(2) & star(3) & alpha & Delta & V & B-V & Sp \\
\hline 84 & 6492 & 1074 & $8: 48: 28.54$ & $12: 03: 58.7$ & 10.59 & 1.12 & G8III \\
\hline 141 & 6485 & 1010 & $8: 48: 38.72$ & $11: 59: 18.6$ & 10.48 & 1.11 & $\begin{array}{l}\text { K2 } \\
\text { III }\end{array}$ \\
\hline 151 & 6494 & 1084 & $8: 48: 42.03$ & $12: 05: 09.0$ & 10.48 & 1.10 & G8III \\
\hline
\end{tabular}

the name of stars have been taken from: (1) - Fagerholm (1906), (2) - Montgomery et al. (1993), (3) - Sanders (1977); the V, B-V and Sp Type data have been adopted from Geller et al. (2015) and Pribulla et al. (2008), respectively.

Table 2. Observations and radial velocities VR $(\mathrm{km} / \mathrm{s})$

\begin{tabular}{|l|l|l|l|l|l|l|l|}
\hline Star & Spec & $\lambda(\AA)$ & date & $\mathrm{VR}_{\text {our }}$ & $\mathrm{VR}_{1}$ & $\mathrm{VR}_{2}$ & $\mathrm{VR}_{3}$ \\
\hline 84 & S34902 & $\begin{array}{l}4500- \\
6000\end{array}$ & $\begin{array}{l}24.04 . \\
2002\end{array}$ & 34.4 & 34.1 & 33.8 & - \\
& & & & & \\
\hline 141 & S39508 & $\begin{array}{l}5275- \\
6765\end{array}$ & $\begin{array}{l}15.04 . \\
2003\end{array}$ & 33.9 & 32.7 & - & 33.0 \\
& & & & & \\
\hline 151 & S39602 & $\begin{array}{l}5275- \\
6765\end{array}$ & $\begin{array}{l}16.04 . \\
2003\end{array}$ & 33.9 & 33.7 & - & - \\
& & & & & \\
\hline
\end{tabular}

$\mathrm{VR}_{1}$ have been taken from Geller et al. (2015); $\mathrm{VR}_{2}$ - from Jacobson et al. (2011); $\mathrm{VR}_{3}$ - from Yong et al. (2005).

Table 3. $\mathrm{T}_{\text {eff }}$ estimates

\begin{tabular}{|l|l|l|l|l|}
\hline Star & $\mathrm{T}_{\text {eff }}$ & $\sigma_{1} \pm$ & $\sigma_{2} \pm$ & $\mathrm{n}$ \\
\hline 84 & 4755 & 28.7 & 64 & 5 \\
\hline 141 & 4755 & 13.0 & 71 & 26 \\
\hline 151 & 4745 & 17.6 & 75 & 18 \\
\hline
\end{tabular}

where $\sigma_{1}$ is SEM, $\sigma_{2}$ is the individual error, $\mathrm{n}$ is the number of relevant calibrations.

Table 4. Surface gravity determinations

\begin{tabular}{|l|l|l|l|l|l|l|}
\hline Star & $\mathrm{T}_{\text {eff }}$ & $\mathrm{Pi}$ & $\mathrm{V}$ & $\mathrm{BC}$ & $\log \mathrm{g}(\mathrm{Pi})$ & $\log \mathrm{g}(\mathrm{IE})$ \\
\hline 84 & 4755 & 0.00097 & 10.59 & -0.433 & 2.25 & 2.7 \\
\hline 141 & 4755 & 0.00088 & 10.48 & -0.433 & 2.12 & 2.7 \\
\hline 151 & 4745 & 0.00240 & 10.48 & -0.439 & 2.98 & 2.8 \\
\hline
\end{tabular}

where $\mathrm{Pi}$ have been adopted from SIMBAD, GAIA collaboration (2016); the Bolometric correction (BC) from Flower (1996).

Table 5. The parameters obtained for the target stars

\begin{tabular}{|l|l|l|l|l|l|}
\hline Star & Spec & $\mathrm{T}_{\text {eff }}$ & $\log \mathrm{g}$ & $\mathrm{Vt}$ & {$[\mathrm{Fe} / \mathrm{H}]$} \\
\hline 84 & 34902 & 4755 & 2.7 & 1.4 & 0.01 \\
\hline 141 & 39508 & 4755 & 2.7 & 1.4 & 0.01 \\
\hline 151 & 39602 & 4745 & 2.8 & 1.3 & 0.04 \\
\hline
\end{tabular}




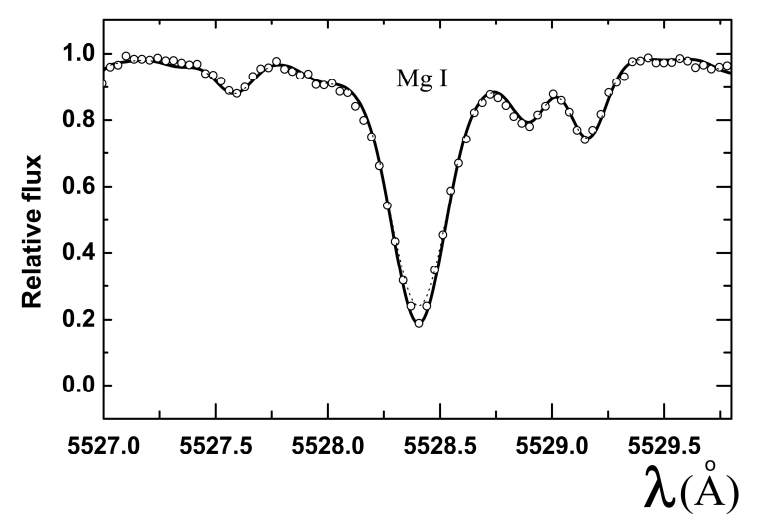

Figure 2: Observed (circles) and calculated (NLTE, solid, and LTE, dashed lines) spectra in the region of $\mathrm{Mg}$ I line for 141 star.

To determine the systematic errors in the elemental abundance resulting from uncertainties in the atmospheric parameter determinations, we derived the elemental abundance of star $141\left(\mathrm{~T}_{\text {eff }} / 4755, \log \mathrm{g} / 2.7, \mathrm{~V}_{\mathrm{t}} / 1.4\right.$, $[\mathrm{Fe} / \mathrm{H}] / 0.01)$ for several models with modified parameters $\left(\delta \mathrm{T}_{\text {eff }}= \pm 50 \mathrm{~K}, \delta \log \mathrm{g}= \pm 0.2, \delta \mathrm{V}_{\mathrm{t}}= \pm 0.1\right)$.

The total uncertainty due to parameter and EW errors for $\mathrm{Fe}$ I, Fe II are 0.06, 0.08, respectively. The determination accuracy varies from 0.06 to 0.15 dex.

A comparison of our data with the results of other authors is given in Table 6 .

In general, there is good agreement between our determinations and those of other authors for all parameters, except for the gravity.

\section{Results and discussions}

We have compared our determinations with those of other authors; as an example, Fig. 5 illustrates the results of the comparison of our data for the star 84 with the data obtained by Tautvaišienè et al., (2000).

The authors have found that the stars 84, 141 and 151 show a slight overabundance of $\mathrm{Na}(+0.17,+0.24$ and +0.22 , respectively) and underabundance of $\mathrm{Zr}(-0.18,-0.19,-18$, respectively).

Sodium. The target three stars have been also studied by Jacobson et al. (2011). For the M 67 stars 84, 141 and 151 the authors have reported slightly different individual values of $[\mathrm{Na} / \mathrm{Fe}]$, such as $-0.06,0.15$, and 0.10 , respectively. According to our LTE determinations, the $\mathrm{Na}$ abundances are $0.03,0.11$, and 0.08 , respectively, these values are fairly similar to the determinations by Jacobson et al. (2011). Yong et al. (2005) have reported an overabundance of $\mathrm{Na} \sim$ +0.30 for the cluster M67. In all the afore-mentioned studies, the $\mathrm{Na} I$ lines $\lambda$ 5682.64, 6154.23 and $6160.75 \AA$ were used for the $\mathrm{Na}$ abundance determinations.

In so doing, despite the difference (about $0.2 \mathrm{dex}$ ) in the sodium abundance obtained by Tautvaišiene et al. (2000) and Jacobson et al. (2011), the equivalent widths EW of the lines 6154.23 and $6160.75 \AA$, e.g., for the star 84 , given in both these paper are similar, the EWs are 84 and $110 \mathrm{~m} \AA$, and 82 and $106 \mathrm{~m} \AA$, respectively.

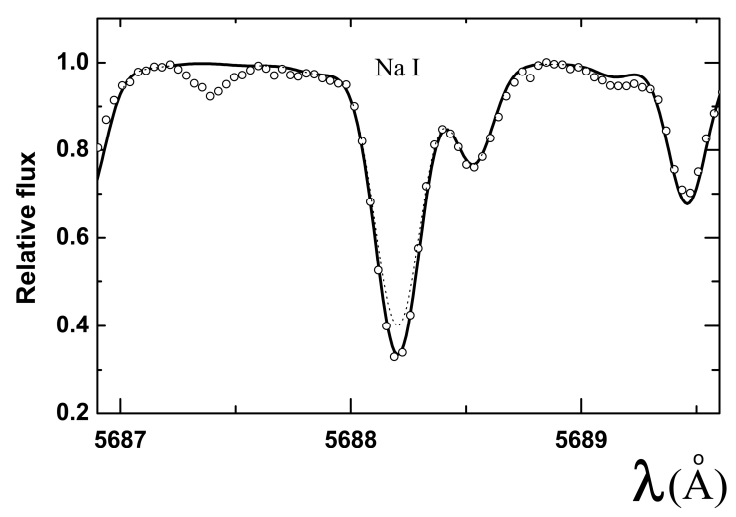

Figure 3: Observed (circles) and calculated (NLTE, solid, and LTE, dashed lines) spectra in the region of $\mathrm{Na}$ I line for 141 star.

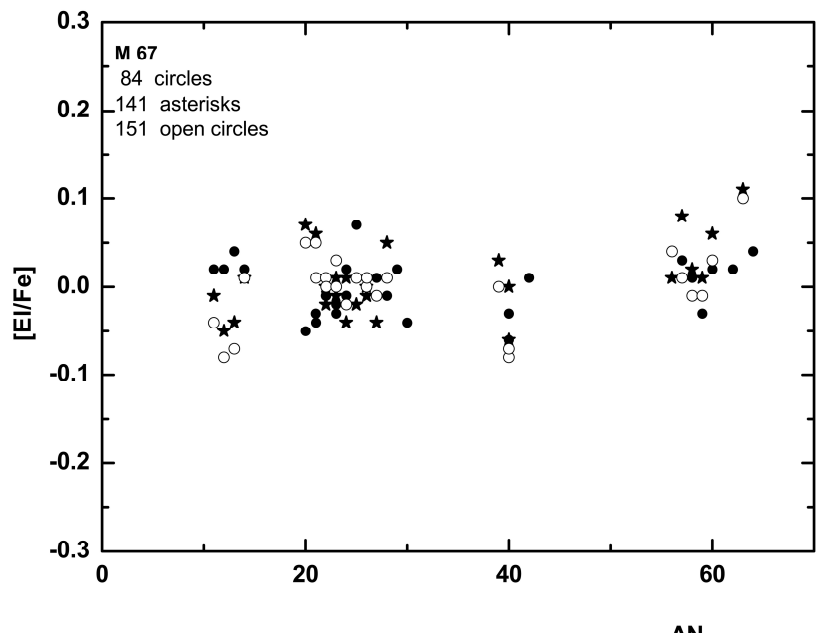

Figure 4: $[\mathrm{El} / \mathrm{Fe}]$ vs. $[\mathrm{Fe} / \mathrm{H}]$ for the target stars

Table 6. A comparison of our findings with the results of other authors

\begin{tabular}{|l|l|l|l|l|l|}
\hline$\#$ & Star & $\mathbf{T}_{\text {eff }}$ & $\log \mathbf{g}$ & {$[\mathbf{F e} / \mathbf{H}]$} & reference \\
\hline 1 & 84 & 4755 & 2.7 & 0.01 & This work \\
\hline & & 4702 & 2.40 & - & Kordoratis et al., 2013 \\
\hline & & - & 2.56 & - & Pace et al., 2012 \\
\hline & & 4650 & 2.5 & 0.00 & Jacobson et al., 2011 \\
\hline & & 4750 & 2.4 & -0.02 & Tautvaisiene et al., 2000 \\
\hline 2 & 141 & 4755 & 2.7 & 0.01 & This work \\
\hline & & 4700 & 2.4 & 0.09 & Jacobson\&Friel, 2013 \\
\hline & & - & 2.56 & - & Pace et al., 2012 \\
\hline & & 4700 & 2.4 & 0.08 & Jacobson et al., 2011 \\
\hline & & 4730 & 2.4 & -0.01 & Tautvaisiene et al., 2000 \\
\hline 3 & 151 & 4745 & 2.8 & 0.04 & This work \\
\hline & & 4740 & 2.62 & - & Meszaros et al., 2013 \\
\hline & & 4700 & 2.4 & -0.02 & Jacobson et al., 2011 \\
\hline & & 4760 & 2.4 & -0.03 & Tautvaisiene et al., 2000 \\
\hline
\end{tabular}




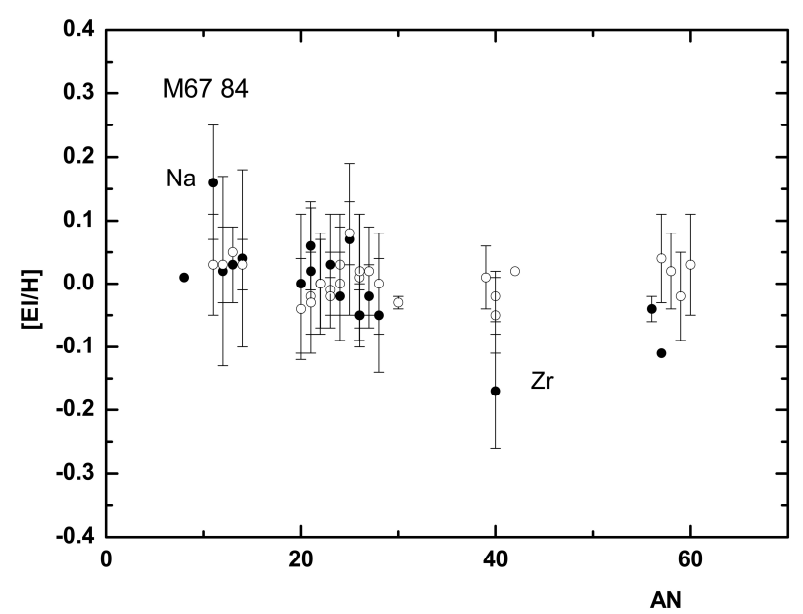

Figure 5: A comparison of our data for the M 67 cluster star F84 (open circles) with those obtained by Tautvaišienè et al. (2000) (full circles).

Zirconium. For the target stars, Jacobson et al. (2011) found underabundance of $\mathrm{Zr}$ equal to $-0.18,-0.16$ and 0.10 , respectively. Later, Jacobson et al. (2013) have redetermined the zirconium abundances and obtained an average value $\langle[\mathrm{Zr} / \mathrm{Fe}]>$ of $-0.01 \pm 0.07$ for the M67 cluster. Yong et al. (2005) have found $[\mathrm{Zr} / \mathrm{Fe}]=-0.28 \pm$ 0.03 for M67; however, Maiorca et al. (2011) have obtained $[\mathrm{Zr} / \mathrm{Fe}]=+0.04 \pm 0.05$. While in the studies by Tautvaišienè et al. (2000), Jacobson et al. (2011, 2013), Yong et al. (2005), as well as in our study, the same Zr I lines, namely $6134.57,6140.46,6243.18,6313.03 \AA$ were used, Maiorca et al. (2011) used the UV Zr II lines, namely 4050.32, 4208.98, $4379.74 \AA$.

According to our $\mathrm{Zr}$ abundance determinations, there is a slight deficit of $\mathrm{Zr}$ for the three target stars: the mean underabundance from $\mathrm{Zr}$ I lines is $-0.05 \pm 0.01$ and from $\mathrm{Zr}$ II lines $<[\mathrm{Zr} / \mathrm{Fe}]>$ is $-0.02 \pm 0.01$.

Thus, in the studied stars in M67, the differences observed in elemental abundance, in particular of sodium and zirconium, depend on the individual approach of different authors and are within the errors of determination.

Now, let us consider the behavior of several elements (Li, Fe, Ba), which are especially manifested in OC's stars.

Lithium. Clump giants have shown $\mathrm{Li}$ abundances slightly lower than the upper limit of the first dredge up (Fig.6). We have only determined the upper limit of the $\mathrm{Li}$ abundance, which is about 0.0 in our stars (141 and 151). These values corroborate the $\mathrm{Li}$ abundance of star 141 (0.0) obtained by Pace et al. (2012). This value corresponds to a greater depletion of $\mathrm{Li}$ during the evolution of stars due to the presence of additional mechanisms that change the lithium abundance and operate inside stars (for example, meridional circulation or diffusion) or are the result of deeper mixing.

The iron problem. Yong et al. (2004) and Shuler et al. (2004) have found remarkable discrepancies between the iron abundances determined with Fe I and Fe II lines in the dwarf stars in the young clusters Hyades, Pleiades and M34. Till now the cause of that is unknown: NLTE effects (Yong et al., 2004) for lines Fe II are negligible; blended lines are not used. We applied the same lines of $\mathrm{Fe} \mathrm{I}$ and $\mathrm{Fe}$ II as in above mention papers and obtained the same iron abundances from lines of both species (within the given accuracy of determinations).

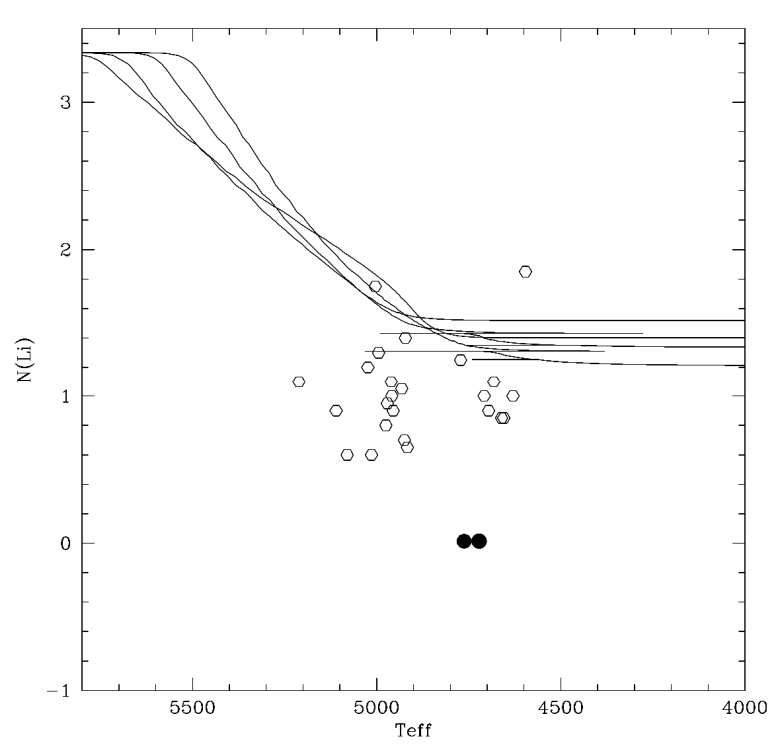

Figure 6: Li abundance vs. $\mathrm{T}_{\text {eff; }}$ our data are marked with full circles; trend lines illustrate the calculations performed by $\mathrm{C}$. Charbonnel for the $\mathrm{Li}$ abundance after the first dredge-up; and the data obtained by Mishenina et al. (2006) for the clump giants are marked with open circles.

The Ba puzzle. The observed $\mathrm{Ba}$ overabundance in young OC's may be due to different causes: 1) additional enrichment with s-process elements from low mass AGB stars (d'Orazi et al., 2009) or with i-process elements (Mishenina et al., 2015); 2) NLTE corrections (Reddy \& Lambert, 2015), but LTE correction is small, <0.05 dex; 3) chromospheric activity or microturbulent velocity $\mathrm{Vt}$ (Reddy \& Lambert, 2017). And if the study of influence of chromospheric activity on $\mathrm{Ba}$ line intensity can be reasonable, then the variation of the turbulent velocity by 3 times for one line, moreover having a depth of line formation of about $\log \tau=-2$ in solar atmosphere, has no substantial grounds. So, there is no definitive answer about the origin of $\mathrm{Ba}$ overabundance for today. Our $[\mathrm{Ba} / \mathrm{Fe}]$ values are from 0.01 to $0.04 \mathrm{dex}$, it is near solar value. M 67 is old cluster and this is the expected result.

\section{Conclusion}

- The results which we have obtained show good agreement with the data reported by other authors.

- Variations in sodium and zirconium abundances are within the given accuracy of determinations.

- The Li abundance determinations suggest a greater depletion of lithium in the course of stellar evolution.

- For M 67 giants, the iron abundances derived from the neutral and ionized iron lines have not shown any significant discrepancies.

- M 67 is found to be an old open cluster with the $[\mathrm{Ba} / \mathrm{Fe}]$ values close to the solar ones.

\section{References}

Carlsson M.: 1986, Uppsala Astron. Obs. Rep., 33.

Carraro G., Girardi L., Bressan A., Chiosi C.: 1996, $A \& A$, 305, 849.

Castelli F., Kurucz R.: 2004, preprint (ArXiv:0405087).

d'Orazi V., Magrini L., Randich S. et al.: 2009, ApJ, 693, L31. 
Flower P. J.: 1975, A\&A, 41, 391.

Fagerholm, E.: 1906, Ueber den Sternhaufen Messier 67 Ph.D. thesis, Uppsala Univ.

Gaia Collaboration: 2016, $A \& A, \mathbf{5 9 5}$, id.A2.

Galazutdinov G.: 1992, Preprint SAO RAS, n 92.

Geller A., Latham D., Mathieu R.: 2015, AJ, 150, id.97.

Jacobson H., Pilachowski C., Friel E.: 2011, $A J$, 142, id.59.

Jacobson H., Friel E.: 2013, AJ, 145, id.107.

Kordopatis G., Gilmore G., Steinmetz M. et al.: 2013, $A J$, 146, id.134.

Korotin S., Andrievsky S., Luck R.: 1999, A\&A, 351, 168.

Kovtyukh V., Soubiran C., Bienaymé O. et al.: 2006, MNRAS, 371, 879.

Kupka F., Piskunov N., Ryabchikova T. et al.: 1999, $A \& A$ Suppl. Ser, 138, 119.

Maiorca E., Randich S., Busso M. et al.: 2011, ApJ, 736, id. 120 .

Mathieu R., Latham D., Griffin R., Gunn, J.: 1986, AJ, 92, 1100.

Mészáros Sz., Holtzman J., García Pérez A. et al.: 2013, $A J$, 146, id.133.

Mishenina T., Bienaymé O., Gorbaneva T. et al.: 2006, $A \& A, 456,1109$.

Mishenina T., Pignatari M., Carraro G. et al.: 2015, MNRAS, 446, 3651.

Montgomery K., Marschall L., Janes K.: 1993, AJ, 106, 181.
Pagel B. E. J.: 1974, MNRAS, 167, 413.

Pace G., Castro M., Meléndez J. et al.: 2012, $A \& A, \mathbf{5 4 1}$, id.A150.

Panchuk V., Klochkova V., Najdenov I., Yushkin M.: 2006, Proc. of the Joint Discuss. n.4 IAU general Assembly of 2006. Ana I. Gómez de Castro and Martin A. Barstow (eds.), 179.

Panchuk V., Klochkova V., Yushkin M. 2017, Astron. Rep., 61, 820.

Pietrukowicz P.; Kaluzny J., Krzeminski W.: 2006, MNRAS, 365, 110.

Pribulla T., Rucinski S., Matthews J. et al.: 2008, MNRAS, 391, 343.

Reddy A., Lambert D.: 2015, MNRAS, 454, 1976.

Reddy A., Lambert D.: 2017, preprint (ArXiv:170707051)

Sanders W. L.: 1977, A\&A Suppl. Ser., 27, 89.

Sarajedini A., Dotter A., Kirkpatrick A.: 2009, ApJ, 698, 1872.

Schuler S., King J., Hobbs L., Pinsonneault M.: 2004, ApJ, 602, L117.

Tautvaišiene G., Edvardsson B., Tuominen I., Ilyin I.: 2000, $A \& A, \mathbf{3 6 0}, 499$.

Taylor B. J.: 2007, AJ, 133, 370.

Tsymbal V.: 1996, ASP Conf. Ser., 108, 198.

Viani L., Basu S.: 2017, preprint (ArXiv:1705.06761).

Yong D., Lambert D., Allende Prieto C., Paulson D.: 2004, ApJ, 603, 697.

Yong D., Carney B., Teixera de Almeida M.: 2005, AJ, 130, 597. 